\title{
APPLICATION OF HM-NETWORKS WITH UNRELIABLE SYSTEMS FOR FINDING THE MEMORY CAPACITY IN THE INFORMATION SYSTEMS
}

\author{
Mikhail Matalytski ${ }^{1}$, Pawel Zajac ${ }^{2}$, Dmitry Kopats ${ }^{3}$ \\ ${ }^{1,2}$ Institute of Mathematics, Czestochowa University of Technology \\ Czestochowa, Poland \\ ${ }^{3}$ Faculty of Mathematics and Computer Science, Grodno State University \\ Grodno, Belarus \\ m.matalytski@gmail.com,pawel_zajac@vp.pl,dk80395@mail.ru
}

Received: 13 November 2017; Accepted: 22 May 2018

\begin{abstract}
To solve the problem of determining the memory capacity of the information systems (IS), it was proposed to use a stochastic model, based on the use of HM (Howard-Matalytski) - queueing networks with incomes. This model takes into account the servicing of requests along with their volumes, the ability to change the volumes of the requests over time and the possibility of damaging IS nodes and their repairs, so servicing of demands can be interrupted randomly. The expressions are generated for the mean (expected) values of total requests volumes in the IS nodes.
\end{abstract}

MSC 2010: 60K20, 60K25, 68M20, $90 B 22$

Keywords: HM-network, information systems, unreliable service, volumes of requests

\section{Introduction}

In IS, the total volume of memory is limited to a certain value, which is usually called memory capacity [1]. When designing systems, the main task is to find the expected volume of memory to take into account the conditions that limit the share of lost information. One of the methods for solving problems of IS design is the use of HM-queueing networks (QN) [2]. According to IS, we will continue to understand systems as information transformation objects, delivered in the form of messages (requests) [1]. HM-networks can be used to determine the volume of a buffer storage of systems that are representing processing IS nodes and transferring demands. Note that this problem is crucial, for example in the design of hubs or communication centers in information networks. The following model can also be used to solve an important problem that recently appeared in the IS, i.e., the problem of excessive buffering (the problem of determining the required 
memory volume) [3]. Bufferbloat - a phenomenon that occurs in packet communication networks, when the buffering causes an excessive increase in the time of the packets and packet delay variation, and the resulting decrease in the throughput volume of IS.

Neglect time dependence of requests processing of their volumes can lead to serious errors in finding the buffer memory in IS. The solution in the general case of the above problems can be based on the use of HM-networks with incomes. In such networks, the request during the transition from one queueing system (QS) to another brings some income last (which is equal to the volume of this request), and the income (volume) of the first QS is reduced by this amount.

Note that the method of finding non-stationary probabilities of states and finding average network characteristics with systems of medium unreliable network systems, operating under high load with the help of multidimensional generating functions are described in the monograph [4], the HM-network with unreliable service $[4,5]$. For the first time, the use of HM-networks to estimate the volume in IS has been described in [6]. In [7, 8], a proposed method of finding the expected volume of homogeneous requests in open network systems with limited waiting times in queues systems, and in [9] - in the HM-network with limited sojourn times of requests.

\section{Finding the expected volumes of requests in the case, when is known only first moments other incomes the transitions between the network states}

Let's look at QN with unreliable QS. Suppose $S_{0}$ handles are reliable, and in other systems $S_{1}, \ldots, S_{n}$ lines can be damaged. The state of the system $S_{i}$ is described by the vector $\left(k_{i}(t), d_{i}(t)\right)$, where $k_{i}(t), d_{i}(t)$ are the number of requests and undamaged lines in the system $S_{i}$ at time $t$, respectively, $0 \leq d_{i}(t) \leq m_{i}, i=\overline{1, n}$. Next, let's specify the $\left(k_{1}(t), d_{1}(t), k_{2}(t), d_{2}(t), \ldots, k_{n}(t), d_{n}(t)\right)$ vector as the state of the network. Assume that the servicing times, the line operating times, and line repair times are independent random variables (RV). The servicing time for IS requests has an exponential distribution with a parameter $\mu_{i}\left(k_{i}(t), d_{i}(t)\right)$; the operation time of each line without damage in this system has an exponential distribution with the parameter $\beta_{i}\left(k_{i}(t)\right), i=\overline{1, n}$. After the damage of the line immediately begins repairing it, the repair time also has an exponential distribution with the parameter $\gamma_{i}\left(k_{i}(t), d_{i}(t)\right), i=\overline{1, n}$. Assume that if the line receives a damage while servicing the call, the request servicing will continue after the line has been repaired. We assume that the discipline of servicing requests in all systems is FIFO. Then at the time interval $[t, t+\Delta t)$, the request can be handled on every line of the system $S_{i}$ with the probability $\mu_{i}\left(k_{i}(t), d_{i}(t)\right) \Delta t+o(\Delta t)$, the line with the probability $\beta_{i}\left(k_{i}(t)\right) \Delta t+o(\Delta t)$ may be damaged or corrected with the probability 
$\gamma_{i}\left(k_{i}(t), d_{i}(t)\right) \Delta t+o(\Delta t), i=\overline{1, n}$. Let us denote by $p_{i j}$ - the probability of passing the request after serving in the $S_{i}$ system to the $S_{j}$ system, $P=\left\|p_{i j}\right\|_{(n+1) \times(n+1)}$ matrix is a matrix of transition probabilities of the irreducible Markov chain.

Let's look at the dynamics of the change in the expected volume of requests in some $S_{i}$ network systems. Let $V_{i}(t)$ be the volume at time $t$, and at the beginning of time the system has the volume $V_{i}(0)=v_{i 0}$. Then this volume at the given QS at time $\Delta t$ can be expressed by the formula

$$
V_{i}(t+\Delta t)=V_{i}(t)+\Delta V_{i}(t, \Delta t),
$$

where $\Delta V_{i}(t, \Delta t)$ - a change in the volume of requests in the system $S_{i}$ in the time interval $[t, t+\Delta t)$. To find this value, you need to find the probabilities of events that may occur at time $\Delta t$ and volume changes in the $S_{i}$ system associated with these events.

1. With probability

$$
1-\left\{\lambda+\sum_{j=1}^{n}\left[\mu_{j}\left(k_{j}(t), d_{j}(t)\right)+\beta_{j}\left(k_{j}(t)\right)+\gamma_{j}\left(k_{j}(t), d_{j}(t)\right)\right] u\left(k_{j}(t)\right)\right\} \Delta t+o(\Delta t)
$$

in the time interval $\Delta t$ the change in system state $S_{i}$ will not take place, the volume of requests in that system increases by $r_{i} \Delta t$, where $E\left\{r_{i}\right\}=c_{i}, i=\overline{1, n}$.

2. With probability $\mu_{i}\left(k_{i}(t), d_{i}(t)\right) u\left(k_{i}(t)\right) p_{i 0} \Delta t+o(\Delta t)$ a request after servicing in $S_{i}$, goes to the external environment, the volume of requests in system $S_{i}$ decreases by the value of $R_{i 0}$, where $E\left\{R_{i 0}\right\}=b_{i 0}, i=\overline{1, n}$.

3. With probability $\lambda p_{0 i} \Delta t+o(\Delta t)$, a request from the outside environment enters the system $S_{i}$ and increases the volume of requests to the size $r_{0 i}, E\left\{r_{0 i}\right\}=a_{0 i}$, $i=\overline{1, n}$.

4. With probability $\mu_{i}\left(k_{i}(t), d_{i}(t)\right) u\left(k_{i}(t)\right) p_{i j} \Delta t+o(\Delta t)$ a request, after servicing in the $S_{i}$ system, goes to the system $S_{j}$; the volume of requests in system $S_{i}$ changes by the value $\Delta V_{i}(t, \Delta t)=-R_{i j}+r_{i} \Delta t$, where $E\left\{R_{i j}\right\}=b_{i j}, i, j=\overline{1, n}, i \neq j$.

5. With probability $\mu_{j}\left(k_{j}(t), d_{j}(t)\right) u\left(k_{j}(t)\right) p_{j i} \Delta t+o(\Delta t)$ a request, after servicing in system $S_{j}$ goes to the system $S_{i}$; the total volume of requests in the system $S_{i}$ will increase by the value of $\Delta V_{i}(t, \Delta t)=r_{j i}+r_{i} \Delta t$, where $E\left\{r_{j i}\right\}=a_{j i}, i, j=\overline{1, n}$, $i \neq j$.

6. With probability $\beta_{j}\left(k_{j}(t)\right) u\left(k_{j}(t)\right) \Delta t+o(\Delta t)$ a number of active lines in system $S_{j}$ decreases by 1 , the volume of requests in the system $S_{i}$ will increase by the value $\Delta V_{i}(t, \Delta t)=r_{i} \Delta t, i, j=\overline{1, n}$. 
The following cases are possible.

7. The number of operating lines in the system $S_{j}$ will decrease by 1 , the request after servicing in the system $S_{i}$ will go to the external environment, $i, j=\overline{1, n}$.

8. The service line in the system $S_{j}$ is damaged, the request enters the system $S_{i}$ from the outside environment, $i, j=\overline{1, n}$.

9. The number of service lines in the system $S_{s}$ will be reduced by 1 , the request after servicing in the system $S_{i}$ will go to the system $S_{j}, i, j=\overline{1, n}, i \neq j$.

10. The number of service lines in the system $S_{s}$ will decrease by 1 , the request after servicing in the system $S_{j}$ will go to $S_{i}, i, j=\overline{1, n}, i \neq j$.

The probabilities of the corresponding events in cases 7-10 are equal $o(\Delta t)$, for example, in case 7 for this probability we have

$$
\begin{aligned}
& {\left[\beta_{j}\left(k_{j}(t)\right) u\left(k_{j}(t)\right) \Delta t+o(\Delta t)\right]\left[\mu_{i}\left(k_{i}(t), d_{i}(t)\right) u\left(k_{i}(t)\right) p_{i 0} \Delta t+o(\Delta t)\right] \times} \\
& \times\left[1-\left\{\lambda+\sum_{\substack{c=1 \\
c \neq i, j}}^{n}\left[\mu_{c}\left(k_{c}(t), d_{c}(t)\right)+\beta_{c}\left(k_{c}(t)\right)+\gamma_{c}\left(k_{c}(t), d_{c}(t)\right)\right] u\left(k_{c} t\right)+\right.\right. \\
& +\left[\mu_{j}\left(k_{j}(t), d_{j}(t)\right)+\gamma_{j}\left(k_{j}(t), d_{j}(t)\right)\right] u\left(k_{j}(t)\right)+ \\
& \left.\left.+\left[\beta_{i}\left(k_{i}(t)\right)+\gamma_{i}\left(k_{i}(t), d_{i}(t)\right)\right] u\left(k_{i}(t)\right)\right\} \Delta t+o(\Delta t)\right]=o(\Delta t) .
\end{aligned}
$$

Changes in the volume of expected requests in the system $S_{i}$ :

$$
\Delta V_{i}(t, \Delta t)=\left\{\begin{array}{c}
-R_{i 0}+r_{i} \Delta t, \text { in case of } 7, \\
r_{0 i}+r_{i} \Delta t, \text { in case of } 8, \\
-R_{i j}+r_{i} \Delta t, \text { in case of } 9, \\
r_{j i}+r_{i} \Delta t, \text { in case of } 10 .
\end{array}\right.
$$

11. With probability $\gamma_{j}\left(k_{j}(t), d_{j}(t)\right) u\left(k_{j}(t)\right) \Delta t+o(\Delta t)$, a number of service lines in system $S_{j}$ will increase by 1 , and the total volume of requests in the system $S_{i}$ by $\Delta V_{i}(t, \Delta t)=r_{i} \Delta t, i, j=\overline{1, n}, i \neq j$.

12. With probability $\gamma_{i}\left(k_{i}(t), d_{i}(t)\right) u\left(k_{i}(t)\right) \Delta t+o(\Delta t)$ one line of service is being repaired in the system $S_{i}$, the total volume of applications will decrease by $\Delta V_{i}(t, \Delta t)=-g_{i}+r_{i} \Delta t$, where $E\left\{g_{i}\right\}=h_{i}, g_{i}-\mathrm{RV}$ with distribution function $H_{i}(x)$ - repair fee for the service line in the system $S_{i}, i=\overline{1, n}$.

In addition, the following cases are possible.

13. There will be a repair of the service line in the system, the request after servicing in the system $S_{j}$ will go to the external environment; $i, j=\overline{1, n}, i \neq j$. 
14. The service line will be repaired in the system $S_{i}$, system $S_{i}$ will go to the outside environment; $i=\overline{1, n}$.

15. The service line will be repaired in the system $S_{j}$, the system $S_{i}$ enters a request from the outside environment; $i, j=\overline{1, n}, i \neq j$.

16. There will be repair of the service line in the system $S_{i}$, and in this system is entering a request from the outside environment, $i=\overline{1, n}$.

17. There will be repair of the service line in the system $S_{s}$, the request after servicing in the system $S_{i}$ will go to system $S_{j}, i, j, s=\overline{1, n}, i \neq s, i \neq j$.

18. The system $S_{i}$ will repair the service line, the request after the servicing system goes to $S_{j}, i, j=\overline{1, n}, i \neq j$.

19. There will be repair of the service line in the system $S_{s}$, the request after servicing system $S_{j}$ goes to $S_{i}, i, j, s=\overline{1, n}, i \neq s, i \neq j$.

20. There will be repair of the service line in the system $S_{i}$, the request after the servicing system $S_{j}$ goes to $S_{i}, i, j=\overline{1, n}, i \neq j$.

21. The system $S_{c}$ will repair the service line, the system will be damaged and the status of the other QS will not change, $i, c, s=\overline{1, n}, i \neq c$.

22. The service line will be repaired in the system $S_{i}$, the service line will be damaged in the system $S_{s}$ and will not change states of other QS, $i, s=\overline{1, n}$.

The probabilities of events taking place in 13-22 cases will be equal $o(\Delta t)$, because, for example, in case 13 we will have

$$
\begin{gathered}
{\left[\gamma_{j}\left(k_{j}(t), d_{j}(t)\right) u\left(k_{j}(t)\right) \Delta t+o(\Delta t)\right]\left[\mu_{i}\left(k_{i}(t), d_{i}(t)\right) u\left(k_{i}(t)\right) p_{i 0} \Delta t+o(\Delta t)\right] \times} \\
\times\left[1-\left\{\lambda+\sum_{\substack{q=1 \\
q \neq i, j}}^{n}\left[\mu_{q}\left(k_{q}(t), d_{q}(t)\right)+\beta_{q}\left(k_{q}(t)\right)+\gamma_{q}\left(k_{q}(t), d_{q}(t)\right)\right] u\left(k_{q}(t)\right)+\right.\right. \\
+\left[\mu_{j}\left(k_{j}(t), d_{j}(t)\right)+\beta_{j}\left(k_{j}(t)\right)\right] u\left(k_{j}(t)\right)+ \\
\left.\left.\left.+\left[\beta_{i}\left(k_{i}(t)\right)+\gamma_{i}\left(k_{i}(t)\right)\right) d_{i}(t)\right] u\left(k_{i}(t)\right)\right\} \Delta t+o(\Delta t)\right]=o(\Delta t) .
\end{gathered}
$$

Changes in the expected volume of requests in the system $S_{i}$ in cases 13-22 are as follows: 


$$
\Delta V_{i}(t, \Delta t)=\left\{\begin{array}{cc}
-R_{i 0}+r_{i} \Delta t, i \neq j, & \text { in case of 13, } \\
-g_{i}-R_{i 0}+r_{i} \Delta t, & \text { in case of 14, } \\
r_{0 i}+r_{i} \Delta t, i \neq j, & \text { in case of } 15, \\
-g_{i}+r_{0 i}+r_{i} \Delta t, & \text { in case of } 16, \\
-R_{i j}+r_{i} \Delta t, i \neq s, j, & \text { in case of 17, } \\
-g_{i}-R_{i j}+r_{i} \Delta t, i \neq j, & \text { in case of } 18, \\
r_{j i}+r_{i} \Delta t, i \neq s, j, & \text { in case of } 19, \\
-g_{i}+r_{j i}+r_{i} \Delta t, i \neq j, & \text { in case of 20, } \\
r_{i} \Delta t, i \neq c, & \text { in case of } 21, \\
-g_{i}+r_{i} \Delta t, & \text { in case of } 22 .
\end{array}\right.
$$

The following cases are possible:

23. The service line will be repaired in the system $S_{c}$, the service line will be damaged in the system $S_{s}$, will not change states of other QS, and after the operation in the system will go to the outside environment, $i, c, s=\overline{1, n}, i \neq c$.

24. The service line will be repaired in the system $S_{i}$, the service line will be damaged in the system $S_{s}$, the request after servicing system goes to the outside environment, $i, s=\overline{1, n}$.

25. The service line will be repaired in the system $S_{c}$, the service line will be damaged in the system $S_{s}$, the request goes into the $S_{i}$ system from the outside environment, $i, c, s=\overline{1, n}, i \neq c$.

26. The service line will be repaired in the system $S_{i}$, the service line will be damaged in the system $S_{s}$, the request goes into the $S_{i}$ system from the outside environments, $i, s=\overline{1, n}$.

27. The service line will be repaired in the system $S_{c}$, the service line will be damaged in the system $S_{s}$, the request after servicing in system $S_{i}$ goes into the $S_{j}, i, j, c, s=\overline{1, n}, i \neq j, i \neq c$.

28. The service line will be repaired in the system $S_{i}$, the service line will be damaged in the system $S_{s}$, the request after servicing in system $S_{s}$ goes into the $S_{j}, i, j, s=\overline{1, n}, i \neq j$.

29. The service line will be repaired in the system $S_{c}$, the service line will be damaged in the system $S_{s}$, the request after servicing in system $S_{j}$ goes into the $S_{i}$, $i, j, c, s=\overline{1, n}, i \neq j, i \neq c$.

30. The service line will be repaired in the system $S_{i}$ and the service line will be damaged in the system $S_{s}$. The request after servicing in system goes into the $S_{i}, i, j, s=\overline{1, n}, i \neq j$. 
In appropriate cases, the probability of 23-30 events are equal $o((\Delta t))^{2}$. For example, in case 23 , the probability will be equal

$$
\begin{gathered}
{\left[\gamma_{c}\left(k_{c}(t), d_{c}(t)\right) u\left(k_{c}(t)\right) \Delta t+o(\Delta t)\right] \times} \\
\times\left[\beta_{s}\left(k_{s}(t)\right) u\left(k_{s}(t)\right) \Delta t+o(\Delta t)\right]\left[\mu_{i}\left(k_{i}(t), d_{i}(t)\right) u\left(k_{i}(t)\right) p_{i 0} \Delta t+o(\Delta t)\right] \times \\
\times\left[1-\left\{\lambda+\sum_{\substack{q=1 \\
q \neq i, c, s}}^{n}\left[\mu_{q}\left(k_{q}(t), d_{q}(t)\right)+\beta_{q}\left(k_{q}(t)\right)+\gamma_{q}\left(k_{q}(t), d_{q}(t)\right)\right] u\left(k_{q}(t)\right)+\right.\right. \\
+\left[\mu_{c}\left(k_{c}(t), d_{c}(t)\right)+\beta_{c}\left(k_{c}(t)\right)\right] u\left(k_{c}(t)\right)+\left[\mu_{s}\left(k_{s}(t), d_{s}(t)\right)+\gamma_{s}\left(k_{s}(t), d_{s}(t)\right)\right] u\left(k_{s}(t)\right)+ \\
\left.\left.+\left[\beta_{i}\left(k_{i}(t)\right)+\gamma_{i}\left(k_{i}(t), d_{i}(t)\right)\right] u\left(k_{i}(t)\right)\right\} \Delta t+o(\Delta t)\right]=o\left((\Delta t)^{2}\right) .
\end{gathered}
$$

We will assume that $\mathrm{RV} R_{i 0}, r_{0 i}, R_{i j}, r_{j i}, g_{i}$, are independent, $i, j=\overline{1, n}, i \neq j$. The changes of the volumes of requests in the system $S_{i}$ in cases 23-30 are equal to:

$$
\Delta V_{i}(t, \Delta t)=\left\{\begin{array}{lc}
-R_{i 0}+r_{i} \Delta t, i \neq c, & \text { in case of } 23, \\
-g_{i}-R_{i 0}+r_{i} \Delta t, & \text { in case of } 24, \\
r_{0 i}+r_{i} \Delta t, i \neq c, & \text { in case of } 25, \\
-g_{i}+r_{0 i}+r_{i} \Delta t, & \text { in case of } 26, \\
-R_{i j}+r_{i} \Delta t, i \neq j, c, & \text { in case of } 27, \\
-g_{i}-R_{i j}+r_{i} \Delta t, i \neq j, & \text { in case of } 28, \\
r_{j i}+r_{i} \Delta t, i \neq j, c, & \text { in case of } 29, \\
-g_{i}+r_{j i}+r_{i} \Delta t, i \neq j, & \text { in case of } 30 .
\end{array}\right.
$$

With constant execution of the process $k(t)$ with $\Delta V_{i}(t, \Delta t)$ can be written

$$
\begin{gathered}
E\left\{\Delta V_{i}(t, \Delta t) / k(t)\right\}=\left(c_{i}+\lambda a_{0 i} p_{0 i}+\sum_{\substack{j=1 \\
j \neq i}}^{n} a_{j i} p_{j i} \mu_{j}\left(k_{j}(t), d_{j}(t)\right) u\left(k_{j}(t)\right)-\right. \\
\left.-\mu_{i}\left(k_{i}(t), d_{i}(t)\right) u\left(k_{i}(t)\right) \sum_{\substack{j=0 \\
j \neq i}}^{n} a_{i j} p_{i j}-h_{i} \gamma_{i}\left(k_{i}(t), d_{i}(t)\right) u\left(k_{i}(t)\right)\right) \Delta t+o(\Delta t), i=\overline{1, n} .
\end{gathered}
$$


Averaging after $k(t)$, taking into account the condition of normalization $\sum_{k} P(k(t)=k)=1$, we will obtain the change in the expected volume of the system $S_{i}$

$$
\begin{aligned}
& E\left\{\Delta V_{i}(t, \Delta t)\right\}=\sum_{k} P(k(t)=k) E\left\{\Delta V_{i}(t, \Delta t) / k(t)\right\}= \\
& =\sum_{k_{1}=0}^{\infty} \ldots \sum_{k_{n}=0}^{\infty} P\left(k(t)=\left(k_{1}(t), \ldots, k_{n}(t)\right)\right) E\left\{\Delta V_{i}(t, \Delta t) / k(t)=\left(k_{1}(t), \ldots, k_{n}(t)\right)\right\}= \\
& =\left(c_{i}+\lambda a_{0 i} p_{0 i}+\left\{\sum_{\substack{j=1 \\
j \neq i}}^{n} a_{j i} p_{j i} \sum_{k} P(k(t)=k) \mu_{j}\left(k_{j}(t), d_{j}(t)\right) u\left(k_{j}(t)\right)-\right.\right. \\
& \left.-\mu_{i}\left(k_{i}(t), d_{i}(t)\right) u\left(k_{i}(t)\right) \sum_{\substack{j=0 \\
j \neq i}}^{n} b_{i j} p_{i j} \sum_{k} P(k(t)=k)\right\}- \\
& \left.-h_{i} \sum_{k} P(k(t)=k) \gamma_{i}\left(k_{i}(t), d_{i}(t)\right) u\left(k_{i}(t)\right)\right) \Delta t+o(\Delta t), i=\overline{1, n} .
\end{aligned}
$$

Let the system $S_{i}$ at the time $t$ have $d_{i}(t), 0 \leq d_{i}(t) \leq m_{i}$. In each of them, the servicing time, the work time and the repair time of damaged lines have an exponential distribution with parameters, $\mu_{i}, \beta_{i}$ and $\gamma_{i}$ respectively, $i=\overline{1, n}$. Assume that the intensity of request servicing and the intensity of repair of damaged lines in the system $S_{i}$ depends linearly on the number of requests and the number of damaged lines.

$$
\begin{gathered}
\mu_{i}\left(k_{i}(t), d_{i}(t)\right) u\left(k_{i}(t)\right)=\left\{\begin{array}{ll}
\mu_{i} k_{i}(t), & k_{i}(t) \leq d_{i}(t), \\
\mu_{i} d_{i}(t), & k_{i}(t)>d_{i}(t),
\end{array}=\mu_{i} \min \left(k_{i}(t), d_{i}(t)\right),\right. \\
\gamma_{i}\left(k_{i}(t), d_{i}(t)\right) u\left(k_{i}(t)\right)=\gamma_{i}\left(m_{i}-d_{i}(t)\right) .
\end{gathered}
$$

We will also assume that the averaging of the expression of $\mu_{i}\left(k_{i}(t), d_{i}(t)\right) u\left(k_{i}(t)\right)$ is $\mu_{i} \min \left(N_{i}(t), \bar{d}_{i}(t)\right)$, i.e. $E\left\{\min \left(k_{i}(t), d_{i}(t)\right)\right\}=\min \left(N_{i}(t), \bar{d}_{i}(t)\right)$.

In addition, $E\left\{m_{i}-d_{i}(t)\right\}=m_{i}-\bar{d}_{i}(t)$, where $N_{i}(t), \bar{d}_{i}(t)$ respectively mean the number of requests (waiting and servicing) and the average number of lines operating in the system $S_{i}$ at the time $t, i=\overline{1, n}$. Given these assumptions, we obtain the approximate 


$$
\begin{gathered}
E\left\{\Delta V_{i}(t, \Delta t)\right\}=\left(c_{i}+\lambda a_{0 i} p_{0 i}+\sum_{\substack{j=1 \\
j \neq i}}^{n} \mu_{j} \min \left(N_{j}(t), \bar{d}_{j}(t)\right) a_{j i} p_{j i}-\right. \\
\left.-\mu_{i} \min \left(N_{i}(t), \bar{d}_{i}(t)\right) \sum_{\substack{j=0 \\
j \neq i}}^{n} b_{i j} p_{i j}-\gamma_{i} h_{i}\left(m_{i}-\bar{d}_{i}(t)\right)\right) \Delta t+o(\Delta t) .
\end{gathered}
$$

Because the operating times and repair lines in the system $S_{i}$ have an exponential distribution, $\gamma_{i}\left(m_{i}-\bar{d}_{i}(t)\right) \Delta t$ - the average number of service lines repaired over time $\Delta t$ in the system $S_{i}, \beta_{i} \bar{d}_{i}(t) \Delta t$ - the average number of service lines in the system $S_{i}$ damaged over time $\Delta t, i=\overline{1, n}$. Therefore $\bar{d}_{i}(t+\Delta t)-\bar{d}_{i}(t)=\gamma_{i}\left(m_{i}-\bar{d}_{i}(t)\right) \Delta t-\beta_{i} \bar{d}_{i}(t) \Delta t$, $i=\overline{1, n}$, where $\Delta t \rightarrow 0$, we get a linear differential equation $\frac{d \bar{d}_{i}(t)}{d t}=\gamma_{i}\left(m_{i}-\bar{d}_{i}(t)\right)-\beta_{i} \bar{d}_{i}(t)$, $i=\overline{1, n}$, and with the initial condition $\bar{d}_{i}(0)=m_{i}$ we have:

$$
\bar{d}_{i}(t)=\frac{m_{i}}{\gamma_{i}+\beta_{i}}\left(\gamma_{i}+\beta_{i} e^{-\left(\gamma_{i}+\beta_{i}\right) t}\right), i=\overline{1, n} .
$$

The equation for the average number of requests in the QS network has the form

$$
\frac{d N_{i}(t)}{d t}=\sum_{\substack{j=1 \\ j \neq i}}^{n} \mu_{j} p_{j i} \rho_{j}(t)-\mu_{i} \rho_{i}(t)+\lambda p_{0 i}, i=\overline{1, n}
$$

It is moved in the same way as in [4] for networks with another feature. The average number of occupied service lines $\rho_{i}(t)$ in the system $S_{i}$ can be approximated by an expression $\rho_{i}(t)=\left\{\begin{array}{l}N_{i}(t), N_{i}(t) \leq \bar{d}_{i}(t), \\ \bar{d}_{i}(t), N_{i}(t)>\bar{d}_{i}(t),\end{array}=\min \left(N_{i}(t), \bar{d}_{i}(t)\right)\right.$. Then the system of equations (4) has the form

$$
\frac{d N_{i}(t)}{d t}=\sum_{\substack{j=1 \\ j \neq i}}^{n} \mu_{j} p_{j i} \min \left(N_{j}(t), \bar{d}_{j}(t)\right)-\mu_{i} \min \left(N_{i}(t), \bar{d}_{i}(t)\right)+\lambda p_{0 i}, i=\overline{1, n},
$$

This is a system of regular linear differential equations with discrete right sides, which can be solved by dividing the phase spaces into a number of areas and finding solutions in each of them. 
From (1), (2) it follows

$$
\begin{gathered}
v_{i}(t+\Delta t)=v_{i}(t)+E\left\{\Delta V_{i}(t, \Delta t)\right\}= \\
=v_{i}(t)+\left(c_{i}+\lambda a_{0 i} p_{0 i}+\sum_{\substack{j=1 \\
j \neq i}}^{n} \mu_{j} \min \left(N_{j}(t), \bar{d}_{j}(t)\right) a_{j i} p_{j i}-\right. \\
\left.-\mu_{i} \min \left(N_{i}(t), \bar{d}_{i}(t)\right) \sum_{\substack{j=0 \\
j \neq i}}^{n} b_{i j} p_{i j}-\gamma_{i} h_{i}\left(m_{i}-\bar{d}_{i}(t)\right)\right) \Delta t+o(\Delta t) .
\end{gathered}
$$

When $\Delta t \rightarrow 0$, we have heterogeneous first order linear equations

$$
\begin{gathered}
\frac{d v_{i}(t)}{d t}=-\mu_{i} \min \left(N_{i}(t), \bar{d}_{i}(t)\right) \sum_{\substack{j=0 \\
j \neq i}}^{n} b_{i j} p_{i j}+\sum_{\substack{j=1 \\
j \neq i}}^{n} \mu_{j} \min \left(N_{j}(t), \bar{d}_{j}(t)\right) a_{j i} p_{j i}- \\
-\gamma_{i} h_{i}\left(m_{i}-\bar{d}_{i}(t)\right)+\lambda a_{0 i} p_{0 i}+c_{i}, i=\overline{1, n} .
\end{gathered}
$$

By setting up the initial conditions $v_{i}(0)=v_{i 0}, i=\overline{1, n}$, you can find the expected volume of network systems.

If the network is functioning in such a way that on average there are no queues in it, ie. $\min \left(N_{i}(t), \bar{d}_{i}(t)\right)=N_{i}(t), i=\overline{1, n}$, the formulas (5) (6) will have the form:

$$
\begin{gathered}
\frac{d N_{i}(t)}{d t}=\sum_{\substack{j=1 \\
j \neq i}}^{n} \mu_{j} p_{j i} N_{j}(t)-\mu_{i} N_{i}(t)+\lambda p_{0 i}, i=\overline{1, n}, \\
\frac{d v_{i}(t)}{d t}=-\mu_{i} N_{i}(t) \sum_{\substack{j=0 \\
j \neq i}}^{n} b_{i j} p_{i j}+\sum_{\substack{j=1 \\
j \neq i}}^{n} \mu_{j} N_{j}(t) a_{j i} p_{j i}-\gamma_{i} h_{i}\left(m_{i}-\bar{d}_{i}(t)\right)+\lambda a_{0 i} p_{0 i}+c_{i}, \\
v_{i}(0)=v_{i 0}, i=\overline{1, n}
\end{gathered}
$$

\section{Expected volumes of requests in central network system}

Let's consider a closed network with a central QS. Let peripheral networks do not have queues on average, ie $\min \left(N_{i}(t), \bar{d}_{i}(t)\right)=N_{i}(t), i=\overline{1, n-1}$, and the central system operates in high load conditions, ie $\min \left(N_{n}(t), \bar{d}_{n}(t)\right)=\bar{d}_{n}(t)$. The system (5) in this case can be rewritten as 


$$
\left\{\begin{array}{l}
\frac{d N_{i}(t)}{d t}=-\mu_{i} N_{i}(t)+\mu_{n} p_{n i} \bar{d}_{n}(t), i=\overline{1, n-1}, \\
\frac{d N_{n}(t)}{d t}=\sum_{i=1}^{n-1} \mu_{i} N_{i}(t)-\mu_{n} \bar{d}_{n}(t) .
\end{array}\right.
$$

The general solution of the system (9) having (3) at the initial conditions, $N_{i}(0)$, $i=\overline{1, n}$, is equal

$$
\begin{gathered}
N_{i}(t)=\alpha_{i}\left(\eta_{i}+\theta_{i} e^{-\left(\gamma_{n}+\beta_{n}\right) t}\right)+\left(N_{i}(0)-\alpha_{i}\left(\eta_{i}+\theta_{i}\right)\right) e^{-\mu_{i} t}, i=\overline{1, n-1}, \\
N_{n}(t)=\sum_{i=1}^{n-1}\left[\left(\alpha_{i}\left(\eta_{i}+\theta_{i}\right)-N_{i}(0)\right) e^{-\mu_{i} t}-\alpha_{i}\left(\eta_{i}+\theta_{i} e^{-\left(\gamma_{n}+\beta_{n}\right) t}\right)\right]+K,
\end{gathered}
$$

where $\alpha_{i}=\frac{\mu_{n} m_{n} p_{n i}}{\gamma_{n}+\beta_{n}}, \eta_{i}=\frac{\gamma_{n}}{\mu_{i}}, \theta_{i}=\frac{\beta_{n}}{\mu_{i}-\gamma_{n}-\beta_{n}}, K=\sum_{i=1}^{n} N_{i}(t)$ - number of requests in the network. For those $N_{i}(t), i=\overline{1, n}$, the system (8) for the expected volume of requests in network systems takes the form

$$
\begin{gathered}
\frac{d v_{i}(t)}{d t}=\mu_{i} b_{i n}\left[\left(\alpha_{i}\left(\eta_{i}+\theta_{i}\right)-N_{i}(0)\right) e^{-\mu_{i} t}-\alpha_{i}\left(\eta_{i}+\theta_{i} e^{-\left(\gamma_{n}+\beta_{n}\right) t}\right)\right]+ \\
+\mu_{n} a_{n i} p_{n i}\left\{\sum_{j=1}^{n-1}\left[\left(\alpha_{j}\left(\eta_{j}+\theta_{j}\right)-N_{j}(0)\right) e^{-\mu_{j} t}-\alpha_{j}\left(\eta_{j}+\theta_{j} e^{-\left(\gamma_{n}+\beta_{n}\right) t}\right)\right]+K\right\}- \\
\frac{d v_{n}(t)}{d t}=\mu_{n} \sum_{j=1}^{n-1} b_{n j} p_{n j}\left(\sum_{i}-\frac{m_{i}}{\gamma_{i}+\beta_{i}}\left(\sum_{i=1}^{n-1}\left[\alpha_{i}\left(\eta_{i}+\theta_{i} e^{-\left(\gamma_{i}+\beta_{i}\right) t}\right)\right)+c_{i}, i=\overline{1, n-1},\right.\right. \\
\left.+\sum_{i=1}^{n-1} \mu_{i} a_{i n}\left[\alpha_{i}\left(\eta_{i}+\theta_{i} e^{-\left(\gamma_{n}+\beta_{n}\right) t}\right)-\left(\alpha_{i}\left(\eta_{i}+\theta_{i}\right)-N_{i}(0)\right) e^{-\mu_{i} t}\right]-K\right\}+ \\
\left.\left.-\gamma_{n} h_{n}\left(m_{n}-\frac{m_{n}}{\gamma_{n}+\beta_{n}}\left(\gamma_{n}+\beta_{n} e^{-\left(\gamma_{n}+\beta_{n}\right) t}\right)\right)+c_{n}\left(\eta_{i}+\theta_{i}\right)\right) e^{-\mu_{i} t}\right]-
\end{gathered}
$$

By integrating these systems with initial conditions, $v_{i}(0)=v_{i 0}, \quad i=\overline{1, n}$, we have 


$$
\begin{gathered}
v_{i}(t)=\mu_{n} a_{n i} p_{n i} \sum_{j=1}^{n-1}\left[\frac{1}{\mu_{j}}\left(N_{j}(0)-\alpha_{j}\left(\eta_{j}+\theta_{j}\right)\right) e^{-\mu_{j} t}+\frac{\alpha_{j} \theta_{j}}{\gamma_{n}+\beta_{n}} e^{-\left(\gamma_{n}+\beta_{n}\right) t}\right]+ \\
+b_{i n}\left[\left(N_{i}(0)-\alpha_{i}\left(\eta_{i}+\theta_{i}\right)\right) e^{-\mu_{i} t}+\frac{\alpha_{i} \mu_{i} \theta_{i}}{\gamma_{n}+\beta_{n}} e^{-\left(\gamma_{n}+\beta_{n}\right) t}\right]-\frac{\gamma_{i} h_{i} \beta_{i} m_{i}}{\left(\gamma_{i}+\beta_{i}\right)^{2}} e^{-\left(\gamma_{i}+\beta_{i}\right) t}+ \\
+\left[\mu_{n} a_{n i} p_{n i}\left(K-\sum_{j=1}^{n-1} \alpha_{j} \eta_{j}\right)-\alpha_{i} \mu_{i} \eta_{i} b_{i n}-\frac{\gamma_{i} h_{i} \beta_{i} m_{i}}{\gamma_{i}+\beta_{i}}+c_{i}\right] t+ \\
+\mu_{n} a_{n i} p_{n i} \sum_{j=1}^{n-1}\left[\frac{1}{\mu_{j}}\left(\alpha_{j}\left(\eta_{j}+\theta_{j}\right)-N_{j}(0)\right)-\frac{\alpha_{j} \theta_{j}}{\gamma_{n}+\beta_{n}}\right]+ \\
+b_{i n}\left[\left(\alpha_{i}\left(\eta_{i}+\theta_{i}\right)-N_{i}(0)\right)-\frac{\alpha_{i} \mu_{i} \theta_{i}}{\gamma_{n}+\beta_{n}}\right]+\frac{\gamma_{i} h_{i} \beta_{i} m_{i}}{\left(\gamma_{i}+\beta_{i}\right)^{2}}+v_{i 0}, i=1, n-1 \\
v_{n}(t)=\mu_{n} \sum_{j=1}^{n-1} b_{n j} p_{n j} \sum_{i=1}^{n-1}\left[\frac{1}{\mu_{i}}\left(\alpha_{i}\left(\eta_{i}+\theta_{i}\right)-N_{i}(0)\right) e^{-\mu_{i} t}-\frac{\theta_{i}}{\gamma_{n}+\beta_{n}} e^{-\left(\gamma_{n}+\beta_{n}\right) t}\right]+ \\
+\sum_{i=1}^{n-1} \mu_{i} a_{i n}\left[\frac{1}{\mu_{i}}\left(\alpha_{i}\left(\eta_{i}+\theta_{i}\right)-N_{i}(0)\right) e^{-\mu_{i} t}-\frac{\theta_{i}}{\gamma_{n}+\beta_{n}} e^{-\left(\gamma_{n}+\beta_{n}\right) t}\right]-\frac{\gamma_{n} \beta_{n} h_{n} m_{n}}{\left(\gamma_{n}+\beta_{n}\right)^{2}} e^{-\left(\gamma_{n}+\beta_{n}\right) t}+ \\
+\left[\mu_{n} \sum_{j=1}^{n-1} b_{n j} p_{n j}\left(\sum_{i=1}^{n-1} \alpha_{i} \eta_{i}-K\right)+\sum_{i=1}^{n-1} \alpha_{i} \mu_{i} \eta_{i} a_{i n}-\frac{\gamma_{n} h_{n} \beta_{n} m_{n}}{\gamma_{n}+\beta_{n}}+c_{n}\right] t+ \\
+\sum_{i=1}^{n-1} \mu_{i} a_{i n}\left[\frac{\theta_{i}}{\gamma_{n}+\beta_{n}}-\frac{1}{\mu_{i}}\left(\alpha_{i}\left(\eta_{i}+\theta_{i}\right)-N_{i}(0)\right)\right]+\frac{\gamma_{n} h_{n} \beta_{n} m_{n}}{\left(\gamma_{n}+\beta_{n}\right)^{2}}+v_{n 0} . \\
b_{n j} p_{n j} \sum_{i=1}^{n-1}\left[\frac{\theta_{i}}{\gamma_{n}+\beta_{n}}-\frac{1}{\mu_{i}}\left(\alpha_{i}\left(\eta_{i}+\theta_{i}\right)-N_{i}(0)\right)\right]+
\end{gathered}
$$

\section{Conclusions}

The article received approximate expressions for finding expected revenues in HM-network systems. These systems can be used when finding the expected memory space in information systems. Further research in this direction may be related to the analysis of arbitrary (non-Markov) random volume registrations and Markov chains applications with other peculiarities, such as limited queue time and faulty service systems. 


\section{References}

[1] Tikhonenko, O. (2006). Metody personalistyczne analizy systemów informacyjnych. Akademicka Oficyna Wydawnicza EXIT.

[2] Matalytski, M., Tikhonenko, O., \& Koluzaeva, E. (2011). Systems and queueing networks: analysis and application [In Russian: Sistemy $i$ seti massovogo obsluzhivanija: analiz i primenenija]. GrSU.

[3] Bufferbloat [Electronic resource]: Wikipedia: http://en.wikipedia.org/wiki/Bufferbloat.

[4] Matalytski, M., \& Statkevich, S. (2014). Stochastic network with bounded waiting time of claims and unrealiable service. [In Russian: Stohasticheskie seti s ogranichennym vremenem ozhidanija zajavok i nenadjozhnym obsluzhivaniem]. GrSU.

[5] Matalytski, M. (2015). Analysis and forecasting of expected incomes in Markov network with bounded waiting time for the claims. Automation and Remote Control, 76(6), 1005-1017.

[6] Matalytski, M., \& Naumenko V. (2015). Zastosowanie HM-sieci kolejkowych dla wyznaczenia objętości pamięci systemów informacyjnych. Studia Informatica, 35(3), 63-69.

[7] Matalytski, M., \& Zajac, P. (2016). Finding of expected volumes of request in systems of the queueing network with limited amount of spaces of expectations. Studia Informatica, 36(1), 89-104.

[8] Matalytski, M., \& Zajac, P. (2017). Expected volumes of requests in systems of the queueing network with a limited number of waiting places. Scientific Issues Jan Dtugosz University in Częstochowa Mathematics XXII, 141-160.

[9] Matalytski M., \& Zajac, P. (2017). Application of HM-networks with impatient claims in finding the memory capacity in the information systems. Journal of Applied Mathematics and Computational Mechanics, 16(3), 47-59. 